\title{
Food for cholera
}

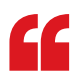

the $V$. cholerae two-component system CrbRS regulates the consumption of acetate g
Infection of the small intestine by Vibrio cholerae causes the severe diarrhoeal disease cholera. The contributions of two virulence factors, the cholera toxin and the toxin coregulated pilus, to intestinal colonization and pathology have been well characterized, but other mechanisms are also thought to be involved in disease pathogenesis. Now, Hang et al. show that the $V$. cholerae two-component system CrbRS regulates the consumption of acetate by this pathogen, which alters insulin signalling and lipid metabolism in the host, resulting in host death.

$V$. cholerae causes a lethal infection in Drosophila melanogaster, which established the fly as a model in which to study host-pathogen interactions. To identify novel genes required for virulence, the authors constructed a library of transposon mutants in a $V$. cholerae strain lacking the cholera toxin gene. By screening this library of mutants for virulence defects, they

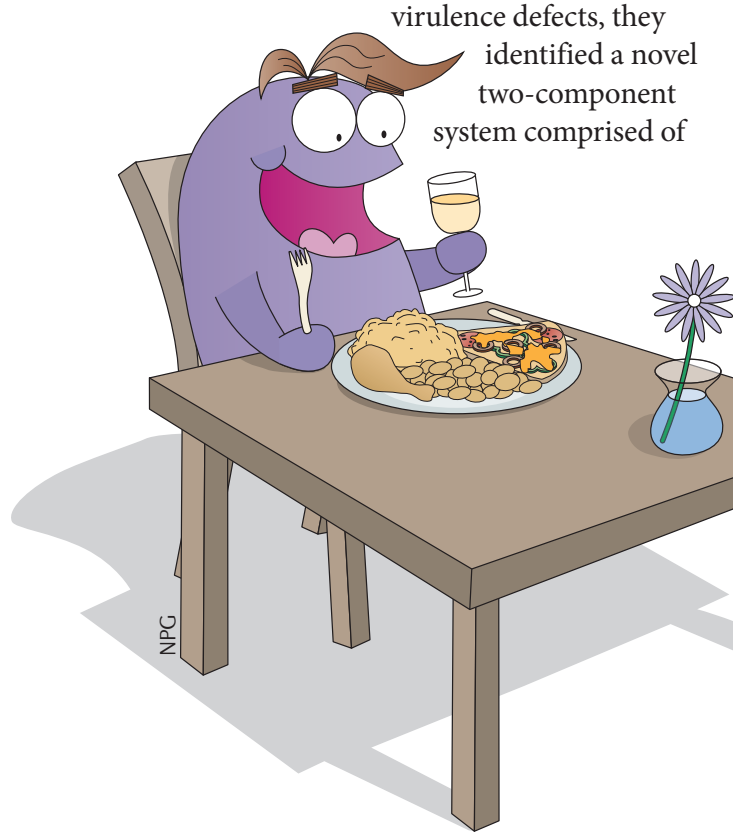

CrbS, a histidine kinase sensor, and CrbR, its cognate response regulator. The authors found that CrbRS controls the acetate switch, a process by which bacteria stop producing acetate and initiate acetate consumption. In Escherichia coli, this switch is solely dependent on transcriptional induction of acs1, the gene encoding acetyl-CoA synthase, which is involved in acetate consumption. Consistent with this, the transcription of acs 1 was reduced more than 1,000 -fold in a $V$. cholerae strain lacking CrbRS, and the deletion of asc 1 reduced $V$. cholerae virulence. Interestingly, deletion of CrbRS or ACS1 had only minor effects on $V$. cholerae colonization of the fly intestine, which suggests that acetate consumption by V. cholerae in the intestine contributes to virulence primarily by affecting the host rather than by promoting bacterial survival.

To understand how acetate consumption by $V$. cholerae contributes to pathogenesis, the authors compared the transcriptional response of D. melanogaster following infection with wild-type and mutant bacteria lacking ACS1 or CrbRS. They observed that wild-type $V$. cholerae inhibits the insulin signalling pathway, whereas the mutant strains do not. Furthermore, infection with wild-type bacteria, but not with the mutant strains, was associated with changes in lipid metabolism in the intestine; lipids accumulated in enterocytes (termed intestinal steatosis), rather than being transported to and stored in adipose tissue. As insulin signalling is known to regulate lipid storage in D. melanogaster, these findings suggest that acetate consumption by $V$. cholerae disrupts insulin signalling, leading to intestinal steatosis, which results in increased host lethality. Notably, removal of lipids from the D. melanogaster diet during infection prevented intestinal steatosis and resulted in increased survival, which indicates that lipid accumulation in enterocytes contributes to the lethality of $V$. cholerae infection.

As the commensal microbiota produces most of the short chain fatty acids in the gut, the authors reasoned that depletion of the microbiota should result in decreased levels of acetate and dysregulation of lipid metabolism, even in uninfected flies. Indeed, germ-free flies displayed decreased insulin signalling, decreased lipid accumulation in adipocytes and increased intestinal steatosis, all of which were reversed by supplementing their diet with acetate.

Together, this study highlights the CrbRS two-component system as a major virulence factor of $V$. cholerae in D. melanogaster that perturbs insulin signalling and lipid metabolism, leading to host death. Interestingly, transcription of the gene encoding CrbS has previously been shown to be activated during $V$. cholerae infection in mice and humans. Therefore, it is possible that a similar process occurs in patients with cholera and future studies are needed to understand whether acetate supplementation can be used as a treatment for cholera infection.

Cláudio Nunes-Alves

ORIGINAL RESEARCH PAPER Hang, S. et al.

The acetate switch of an intestinal pathogen disrupts host insulin signaling and lipid metabolism. Cell Host Microbe 16, 592-604 (2014) 